\title{
Incidents in Anaesthesia: Past Occurrence and Future Avoidance
}

\section{"I do not want two diseases- one nature made - One doctor made"(Napoleon Bonaparte (1769-1821) ${ }^{1}$}

The concept of medical error and iatrogenic injury or adverse events in the healthcare sectors are not a new phenomenon and are increasingly becoming a cause for concern. It has been recognized for thousands of years that attempts to improve health and reduce suffering could themselves be associated with harmful effects to the patients. It constitutes the basis of the Hippocratic Oath "primum non nocere"-or "first do not harm" first enunciated over 2000 years ago. Estimates derived from UK, USA and Australian studies indicate that adverse events are associated with about $10 \%$ of all hospital admission and account in direct medical costs for $5 \%$ of the total health budget ${ }^{2}$. Incident and accident causation can be seen to arise from complex, dynamic interaction between organizational, workplace and personal factors. Personal factors include slips, lapses, rule - and knowledge-based mistakes and violation. Latent failures in the system also contribute to the generation of organizational and individual accidents. An incident is defined as any event which affected or could have affected the safety of the patient or which either caused harm or if uncorrected might have caused harm to a patient while under anaesthesia care ${ }^{3}$. On most occasions the incident is simple, if not detected and corrected, it can evolve into a critical incident and the potential for a significant negative outcome arises. When accidents occur they must be investigated not with the goal of apportioning blame but as a means of finding the chains of events and contributory factors that lead to it, in order to prevent further occurrences. Such an investigations will reveal gaps and inadequacies in the health care system. Furthermore, because it is a proactive activity not a mortality review, it is more attractive, forward looking and should be encouraged ${ }^{4}$.Patient safety has been highlighted more recently by widely publicized investigations at local, national and international levels. It is now obvious that doctors will be held much more accountable for their action in the future than they have been to date.
Critical incident investigation was first used in the 1940s described by Flanagan during World War IIas a technique to improve safety and performance among military pilots ${ }^{5}$. In 1978, Cooper and Colleagues used what they described as a "modified critical incident technique" to interview anesthetists and obtain description of preventable incident ${ }^{6}$. It is now common place for the department of anaesthesia to record and discuss adverse incidents and near misses with a view to learning from the problems encountered and preventing their re-occurence.However the knowledge of and learning from these accidents tend to shared only at a local level and subsequent improvement in patient safety thus remain local. In order to share and expand learning more widely at a national and international level, a number of different critical incident reporting systems have been set up in different countries ${ }^{7}$. In Australia, the Australian Incident Monitoring Study begins in the late 1980 s as an anaesthesia specific venture $^{8}$. An anaesthesia-specific on line reporting system has been operating in Switzerland since the mid $1990 \mathrm{~s}^{9}$ and more recently, the German Society of Anaesthesiology and Intensive Care has set up its own Patient Safety Optimisation System ${ }^{10}$. Both these sites offer the opportunity to report incidents and read those posted by others. Denmark also has a nationally conceived Patient Safety Database to which reports can be uploaded, although this is not specific to anaesthesia ${ }^{11}$. In the UK, the Royal College of anaesthestists has consistently encouraged in incident reporting in anaesthesia. The UK National patient Safety Agency, established in 2001, set up a Reporting and Learning system to collect and learn from adverse incidents and near misses ${ }^{12}$.Sri Lanka introduced an anaesthetic incident reporting system in 2010. It is the college of anaesthesiologists of Sri Lanka that co-ordinates the incidence reporting ${ }^{13}$. The incidence report technique is very simple; it involves the anonymous reporting of any incident that occurs during an anaesthetic. One would need to design a form on which the incident can be reported. The form used is based to a great extent on the form used in Australian Incident Monitoring System 
modified to suit local circumstances. It successfully highlighted weakness where procedural changes have been able to prevent repetition.

Modern anaesthesia is very safe but problems and mistakes do occur often with no consequences for the patient. Anesthesiologists working environment is characterized by high dynamism and uncertainty, time pressure, ill formed problems, complex humans machine interactions and risk. Wherever humans work, failures are inevitably do occur ${ }^{14}$. The errors are often identifiable and repetitive, so they can be analyzed and classified ${ }^{15}$. At present significant problems remain with local and national incident reporting system. These includes the fear of fault finding action, poor safety culture in an organization, lack of understanding among clinicians about what should be reported, lack of awareness of how the reported incidences will be analysed, how will the reports ultimately lead to change which will improve the safety. In lack of systemic analysis of reports and feedback directly to the clinicians are seen as major barriers to the clinical engagement. The success of any new critical incidents reporting and monitoring systems will depend upon making sure that the system is simple, unambiguous, userfriendly, and intuitive ${ }^{16}$.

It is our sincere hope that the new venture will bring great benefits to our speciality. We believe that the professions of anaesthesia shoud develop such a culture, where incident reporting is a routine occurrence. The partnership will harness the enthusiasm of the profession for reporting threat to patient safety and acting to eliminate them. Finally, it will not be too optimistic to speculate that, as in many other areas, specialityspecific national incident reporting in anaesthesia will be a model for future initiatives in other specialties. Thus critical incident reporting should be introduced in all anaesthesia departments as a part of quality assurance programs to ensure improved patient care as an educational tool but never as a punititive measure ${ }^{17}$.

"It takes a long time to bring excellence to maturity."(Pubilius Syrus 100BC) ${ }^{7}$

\section{Md. Abdul Hye}

Professor, Dept. of Anaesthesia, Analgesia and Intensive Care Medicine, BSMMU, Dhaka.

\section{References:}

1. Cited in Laurence DR, Bennett PN (eds). Clinical Pharmacology, London: Churchill Livingstone, 1995

2. Kluger MT, Runciman WB. Incident monitoring in anaesthesia.In Healy TEJ, Knight PR (eds) Wylie and ChurchillDavidson's A Practice of Anesthesia. $7^{\text {th }}$ edn. London: Arnold, 2003; 684

3. James RH.1000 anaesthetic incidents: experience to date. Anaesthesia. 2003; 54:456-463

4. Amucheazi AO., Ajuzieogu OV. Critical incident during anaesthesia in a developing country: A retrospective audit. Anaesthesia: Essays and Researches.2010; 4:2:64-9

5. Flanagan JC. The critical incident technique. Psychol Bull 1954; 51:327-58

6. Cooper JB, Newblower RS, Long CD, McPeek B. Preventable anaesthesia mishaps: a study of human factors. Anesthesiology 1978; 49:399-406

7. Smith A.F, Mahajan RP. National Critical incident reporting: improving patient safety. Br J Anaesth 2009; 103:623-5

8. Merry AF. Safety in anesthesia: reporting incidents and learning from them, Anaesthesia 2008; 63:337-9

9. Available from http://www.medana.unibas.ch/cirs

10. Available from http://www.pasos-ains.de/ indexxSSL.php

11. Available from http://www.dpsd.dk/

12. Rollin A-M. Critical incident reporting 2001.R Coll Anaesthetists Bull 2001; 9:413-4

13. Jayasuria j. Establishing an incident reporting system. Conference lectures. $9^{\text {th }}$ congress of SARRC. Association of anaesthesiologists, 2011: 204

14. Runciman WB.etal. The Australian Incident Monitoring Study: Errors,incidents and accidents in anesthetics practice. Anaesthesia Intensive Care. 1993; 21: 506-19

15. Allnut MF. Human factors in accidents. $\mathrm{Br} \mathrm{J}$ Anaesth1987; 59:856-6.

16. Secker-Walker J, Taylor-Adams S. Critical incident reporting. In: Vincet C, ed. Clinical Risk Management Enhancing Patient Safety. London: BMJ Books, 2001; 41

17. Choy YC. Critical incident monitoring in anesthesia. Med J Malaysia. 2006; 61:577-85 